woraus unter Berücksichtigung von (I) folgt:

$$
a \sin \not p_{1} \frac{\sin z_{1}}{\sin w_{1}}=c \sin q_{1}
$$

Setzt man ferner $A S=p$, so gilt:

$$
\sin v \sin p=\sin v_{1} \sin p_{1}
$$

und durch Differentiation :

$$
\left(v_{1}-v\right) \operatorname{ctg} v_{1}+\left(p_{1}-\not p\right) \operatorname{ctg} p_{1}=0 .
$$

Indem man dies alles in den letzten Ausdruck von $m$ einsetzt, ergiebt sich:

$$
m=\frac{c}{V} \sin q_{1}\left[\mathbf{1}+\operatorname{ctg} p_{1}\left(p_{0}-p\right)\right]
$$

Bedenkt man nun noch, dass

$$
p_{0}-p=-l=-\frac{a}{V} \sin p_{0}
$$

ist, wofür man hier $-\frac{a}{V} \sin p_{1}$ einsetzen darf, so bildet sich :

$$
m=\frac{c}{V} \sin q_{1}\left[\mathbf{I}-\frac{a}{V} \cos p_{1}\right]
$$

Die Grösse $c \sin q_{1}$ ist die alleinige Wirkung der jährlichen Aberration, während die Klammer die von Villarceau gefundene Abhängigkeit der Aberrationsconstante vom Sternort darstellt. $\mathrm{Da}$ die letztere nach den bisherigen Erfahrungen verschwindend ist, kann man behaupten, dass die gebräuchlichen Aberrationsformeln den scheinbaren Ort $S_{1}$ streng in den Ort $S_{0}$ überführen. Dies ist dann der Ort, den unsere Cataloge geben, den man für Praecession und Nutation corrigirt und dessen restirende Veränderlich. keit man als Eigenbewegung auffasst.

Um wahre Oerter zu erhalten, hätte man an die Catalogörter nun noch die Verschiebungen $l$ nach (4) anzubringen. Wie man aus (4) sieht, hängt $l$ nur von der relativen Lage der Punkte $A$ und $S$ ab und ist daher constant, so lange $S$ keine Eigenbewegung besitzt. In diesem Fall ist auch der Catalogort $S_{0}$ ohne Eigenbewegung. Für einen bewegten Stern dagegen seien $S$ und $S^{\prime}$ wahre Oerter zu zwei verschiedenen Zeiten und $S_{0}$ und $S_{0}^{\prime}$ die zugehörigen Catalogörter. Man hat dann aus der Figur, wenn $A S=p$ und $A S^{\prime}=p^{\prime}$ ist:

München I 894 März 28.

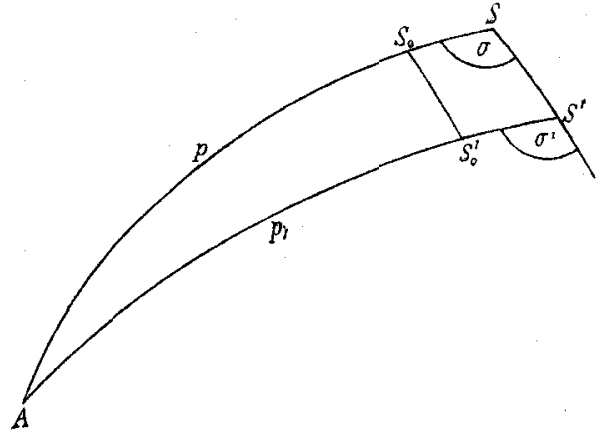

$\sin p \sin \sigma=\sin p^{\prime} \sin \sigma^{\prime}$

Da man ferner das Viereck $S S^{\prime} S_{0} S_{0}{ }^{\prime}$ als eben betrachten darf und hinreichend genau

$$
S_{0} S=\frac{a}{V} \sin p, \quad S_{0}{ }^{\prime} S^{\prime}=\frac{a}{V} \sin p^{\prime}
$$

ist, so sagt diese Gleichung nichts anderes, als dass $S_{0} S_{0}{ }^{{ }^{\prime}}$ parallel $S S^{\prime}$ ist, d. h. die Richtung der Eigenbewegung bleibt unverändert. Was ihre Grösse anbetrifft, so liefert eine leichte Rechnung :

$$
S_{0} S_{0}{ }^{\prime}=S S^{\prime}\left[\mathbf{1}-\frac{a}{V} \cos p\right]
$$

Die Eigenbewegungen multipliciren sich also mit demselben Factor wie die Aberrationsconstante, der hier mit noch grösserem Recht als oben gleich Eins gesetzt werden darf. Demnach folgt, dass unsere Cataloge die wahren Eigenbewegungen geben.

Als einzige Wirkung der Aberration bleibt eine constante Verzerrung des Himmelsgewölbes übrig, auf deren Beseitigung man stets verzichten wird. Sollte sich später einmal eine Verschiedenheit der Aberrationsconstanten von Stern zu Stern bemerkbar machen, so hätte man nur den Villarceau'schen Factor an Aberrationsconstante und Eigenbewegung anzubringen.

Producte der Praecession und der saecularen Aberration können, wie Herr Dr. Battermann Astr. Nachr. Bd. I 8 bemerkt hat, schon deshalb nicht auftreten, weil $l$ nur von der relativen Lage von Stern und Apex, nicht aber von der Wahl eines Coordinatensystems abhängt.

\title{
Ueber die Identität der Lambert'schen und Olbers'schen Methode zur Berechnung parabolischer Bahnen.
}

\section{Von Robert Vogel.}

Es ist bekannt, dass mehrere wichtige Entdeckungen von verschiedenen Personen fast gleichzeitig gemacht wurden. Dass so etwas in Bezug auf Thatsachen vorkommen kann, ist sehr leicht verständlich, aber viel seltener trifft es ein, dass zwei Verfasser nicht gleichzeitig und dennoch unabhängig von einander zwei identische Methoden zur Lösung einer gewissen Aufgabe aufstellen. Das letztere war der
Fall mit der Methode, welche von Lambert (Johann Heinrich) im Jahre 1771 und von Olbers im Jahre r797, also ungefähr ein Vierteljahrhundert später, gegeben wurde.

Vor sechs Jahren fiel mir diese Thatsache beim Isesen des Lambert'schen Mémoire's auf und ich veröffentlichte damals einen Aufsatz in russischer Sprache, worin ich unter anderem auch auf die Identität beider Methoden hinwies; 
da aber die Thatsache an und für sich von Interesse ist und bis jetzt nicht allgemein bekannt zu sein scheint, so wird folgender kurzer Auszug nicht überflüssig sein.

In den Nouveaux Mémoires*) der Berliner Akademie, in dem Aufsatz „Observations sur l'orbite apparente des comètes giebt Lambert für das Verhältniss der (nicht curtirten) Distanzen die Formel

$$
\frac{\Delta_{2}}{\Delta}=x=\frac{\tau}{t} \cdot \frac{\sin \omega}{\sin \varphi}
$$

Hier sind $\tau$ und $t$ die Zwischenzeiten, d. h. in der jetzt üblichen Bezeichnung $t_{2}-t_{1}$ und $t_{1}-t$. Wenn man den ersten geocentrischen Ort des Cometen $K$ mit dem dritten $K_{2}$ und den zweiten Ort $K_{1}$ mit dem entsprechenden
Sonnenorte $S_{1}$ durch Bogen grösster Kreise verbindet, so ist, wenn $O$ den Durchschnitt beider Bogen bezeichnet, $\omega=O K ; \varphi=O K_{2}$.

Zur Berechnung der Distanzen selbst empfiehlt Lambert die Gleichung (I) in Verbindung mit der folgenden durch Versuche aufzulösen.

$$
T=\frac{(S M+S N+M N)^{3 / 2}-(S M+S N-M N)^{3 / 2}}{\text { 1 } 2 m}
$$

d. h. in der jetzt gebräuchlichen Bezeichnung

$$
6 k\left(t_{2}-t\right)=\left(r+r_{2}+c\right)^{3 / 2}-\left(r+r_{2}-c\right)^{3 / 2}
$$

Es ist leicht zu beweisen, dass die Lambert'sche Formel (I) mit der Olbers'schen

$$
\frac{\varrho_{2}}{\varrho}=M=\frac{t_{2}-t_{1}}{t_{1}-t} \cdot \frac{\left(\operatorname{tg} \beta_{1} \sin \left(\lambda-L_{1}\right)-\operatorname{tg} \beta \sin \left(\lambda_{1}-L_{1}\right)\right)}{\left(\operatorname{tg} \beta_{2} \sin \left(\lambda_{1}-L_{1}\right)-\operatorname{tg} \beta_{1} \sin \left(\lambda_{2}-L_{1}\right)\right)}
$$

identisch ist, wo $\beta \beta_{1} \beta_{2} \lambda \lambda_{1} \lambda_{2}$ die Breiten und Längen des Cometen und $L_{1}$ die zweite Sonnenlänge ist.

Zu diesem $Z$ weck bezeichne man die Neigungswinkel der Kreise $S_{1} K, S_{1} K_{1}, S_{1} K_{2}$ zur Ekliptik durch $\gamma \gamma_{1} \gamma_{2}$, dann hat man:

$$
\sin \left(S_{1} K\right) \sin \gamma=\sin \beta ; \quad \sin \left(S_{1} K_{1}\right) \sin \gamma_{1}=\sin \beta_{1} ; \sin \left(S_{1} K_{2}\right) \sin \gamma_{2}=\sin \beta_{2}
$$

$\sin \left(S_{1} K\right) \cos \gamma=\cos \beta \sin \left(\lambda-L_{1}\right) ; \quad \sin \left(S_{1} K_{1}\right) \cos \gamma_{1}=\cos \beta_{1} \sin \left(\lambda_{1}-L_{1}\right) ; \sin \left(S_{1} K_{2}\right) \cos \gamma_{2}=\cos \beta_{2} \sin \left(\lambda_{2}-L_{1}\right)$

$$
\begin{aligned}
& \sin \omega \sin \left(S_{1} O K\right)=\sin \left(S_{1} K\right) \sin \left(\gamma-\gamma_{1}\right) \\
& \sin \varphi \sin \left(S_{1} O K_{2}\right)=\sin \left(S_{1} K_{2}\right) \sin \left(\gamma_{1}-\gamma_{2}\right)
\end{aligned}
$$

Daraus folgt

$$
\frac{\sin \omega}{\sin \varphi}=\frac{\sin \left(S_{1} K\right) \sin \left(\gamma-\gamma_{1}\right)}{\sin \left(S_{1} K_{2}\right) \sin \left(\gamma_{1}-\gamma_{2}\right)}
$$

Schafft man die Winkel $\gamma \gamma_{1} \gamma_{2}$ fort, so kann der Gleichung (I) die folgende Form gegeben werden:

$$
\frac{x \cos \beta_{2}}{\cos \beta}=\frac{\left(t_{2}-t_{1}\right)}{\left(t_{1}-t\right)} \cdot \frac{\left(\operatorname{tg} \beta_{1} \sin \left(\lambda-L_{1}\right)-\operatorname{tg} \beta \sin \left(\lambda_{1}-L_{1}\right)\right)}{\left(\operatorname{tg} \beta_{2} \sin \left(\lambda_{1}-L_{1}\right)-\operatorname{tg} \beta_{1} \sin \left(\lambda_{2}-L_{1}\right)\right)}
$$

Auf diese Weise ist die Lambert'sche Formel (r) auf die Olbers'sche (3) zurückgeführt, denn $\frac{x \cos \beta_{2}}{\cos \beta}$ ist das Verhältniss der curtirten Distanzen. $\dagger$ )

Da die Gleichungen (2) und (3) die Fundamentalgleichungen der Olbers'schen Methode bilden, so ist also letztere mit der Lambert'schen Methode identisch.

Kiew 1894 Juli 6.

\section{Robert Vogel}

Docent an der Universität zu Kiew.

*) Nonveaux Mémoires de l’Académie Royale des Sciences et Belles-lettres. Année I 771. Berlin I773, page 363 .

t) Ich nehme oben diejenige Form des Ausdruckes für $M$, welche bei numerischen Berechnungen gewöhnlich angewandt wird. Wollte man von derjenigen ausgehen, die Olbers im $\$ 36$ seiner Abhandlung giebt, so wäre die Identität beider Methoden fast auf der Stelle klar.

\section{On the Harvard Photometric Observations.}

\section{By S. C. Chandler.}

In his article in No. 3229 , Prof. Pickering acknowledges the existence of all the errors specified in No. 3214 , except the case of $\mathrm{V}$ Coronae, where $\mathrm{I}$ had myself indicated the possible but not probable escape of which he avails himself. He also admits the correctness of all my surmises as to the stars erroneously observed for the variables, except one, where his mis-identification was with a star a trifle further off. We may therefore concentrate attention entirely upon the necessary inferences to be drawn from the nature of the errors.

It would seem that no careful astronomer who had occasion to attempt to use these photometric observations, and who had found such an appalling ratio of certain mistake as fifteen out of eighty six possible cases, and of reasonably probable mistake in a much higher proportion, could have failed to arrive at the conclusion that $\gg$ it 